\title{
Measurements Obtained From Esophageal Balloon Catheters Are Affected by the Esophageal Balloon Filling Volume in Children With ARDS
}

\author{
Justin C Hotz RRT-NPS, Cary T Sodetani RRT-NPS, Jeffrey Van Steenbergen RRT-NPS, \\ Robinder G Khemani MD MSCI, Timothy W Deakers MD PhD, and \\ Christopher J Newth MD FRCPC
}

\begin{abstract}
BACKGROUND: Esophageal balloon inflation volume may affect the accuracy of transpulmonary pressure estimates in adults, but the effect is unknown in pediatrics. Using a combination bench and human study, we sought to determine a range of optimal filling volumes for esophageal balloon catheters and to derive a technique to inflate catheters to yield the most accurate estimates of pleural pressure. METHODS: In the laboratory study, we evaluated 4 pediatric and adult esophageal balloon catheters, a liquid-filled catheter, and a micro-tip catheter, both with and without a model esophagus. We compared the measured esophageal pressure for each type of catheter within a pressurized chamber. Esophageal balloon catheters were also tested by manipulating the esophageal balloon inflation volume, and we attempted to derive a filling-volume technique that would assure accuracy. We then tested the feasibility of this technique in 5 mechanically ventilated pediatric subjects with ARDS. RESULTS: In the laboratory study, smaller inflation volumes underestimated the chamber pressure at higher chamber pressures, and larger inflation volumes overestimated the chamber pressure at lower chamber pressures. Using an optimal filling-volume technique resulted in a mean total error that ranged from -0.53 to $-0.10 \mathrm{~cm} \mathrm{H}_{2} \mathrm{O}$. The optimal filling-volume values for the pediatric catheters were $0.2-0.6 \mathrm{~mL}$, and $0.4-0.8 \mathrm{~mL}$ for the adult catheters. When correctly positioned and calibrated, the micro-tip transducer and liquid-filled catheters were within $\pm 1 \mathrm{~cm} \mathrm{H}_{2} \mathrm{O}$ of chamber pressure for all ranges of pressure. In the clinical study, high variability in measured esophageal pressure and subsequent transpulmonary pressure during exhalation and during inhalation was observed within the manufacturer's recommended esophageal balloon inflation ranges. CONCLUSIONS: Manufacturer-recommended esophageal balloon inflation ranges do not assure accuracy. Individual titration of esophageal balloon volume may improve accuracy. Better esophageal catheters are needed to provide reliable esophageal pressure measurements in children. Key words: esophagus; catheters; lung; pressure; pleura; respiration; artificial; respiratory distress syndrome; adult; pediatrics. [Respir Care 2018;63(2):177-186. (C) 2018 Daedalus Enterprises]
\end{abstract}

\section{Introduction}

During mechanical ventilation, transpulmonary pressure $\left(\mathrm{P}_{\mathrm{L}}\right)$ is estimated as the airway pressure $\left(\mathrm{P}_{\mathrm{aw}}\right)$ (as a surro-

Messrs Hotz, Sodetani, and Van Steenbergen are affiliated with the Department of Respiratory Care, Children's Hospital Los Angeles, CA. Drs Khemani, Deakers, and Newth, as well as Mr Hotz, are affiliated with the Department of Anesthesiology and Critical Care Medicine, Children's Hospital Los Angeles, CA. Drs Khemani, Deakers, and Newth are also gate for alveolar pressure) minus the esophageal pressure $\left(\mathrm{P}_{\mathrm{es}}\right)$ (as a surrogate for pleural pressure). ${ }^{1}$ Using $\mathrm{P}_{\mathrm{L}}$ to guide ventilator management has theoretical benefits in both adult and pediatric patients with ARDS to prevent

\footnotetext{
affiliated Department of Pediatrics, Keck School of Medicine, University of Southern California, Los Angeles.

Supplementary material related to this paper is available at http:// www.rcjournal.com.
} 


\section{Measurements in Pediatric Esophageal Balloon Catheters}

ventilator-induced lung injury from atelectatrauma and barotrauma. ${ }^{2-7}$ It is generally accepted that $P_{a w}$ is reflective of alveolar pressure when flow is interrupted (ie, inspiratory and expiratory holds), although this may not always be the case, particularly when some alveoli are closed. ${ }^{1}$ Pleural pressure is also difficult to measure directly in the clinical setting, so it is often estimated with $\mathrm{P}_{\mathrm{es}}$ using an air-filled esophageal balloon catheter. ${ }^{8}$ However, the accuracy of esophageal balloon catheters may vary on the basis of body position, intrathoracic pressure, lung volume, and the elastance of the esophageal balloon and esophagus. ${ }^{2,9,10}$ Previous bench and clinical studies using adult balloon catheters have demonstrated that small changes to balloon inflation volume, even within the manufacturer's suggested range, can affect the reliability of measurements. ${ }^{11-13}$ While some pediatric-specific balloon catheters exist, data regarding their accuracy as a function of inflation volume are lacking. Using a combination bench and clinical study, we endeavored to determine the accuracy of esophageal balloon catheters as a function of inflation volume, to derive and validate a method to determine the optimal balloon inflation volume for a given subject (in vivo calibration method), and to explore the accuracy of other methods to measure $\mathrm{P}_{\mathrm{es}}$, such as liquidfilled catheters or fiberoptic transducers.

\section{Methods}

Our methods involved a laboratory component, followed by a clinical component in children with ARDS. For the laboratory component, we compared the accuracy of $\mathrm{P}_{\mathrm{es}}$ in adult and pediatric esophageal balloon catheters using a controlled pressure chamber across a range of positive pressures at various esophageal balloon inflation volumes, both within and below the manufacturer-recommended ranges. During the laboratory part of the study, we developed and validated a technique to identify the filling volume of the balloon that resulted in the most accurate mea-

A portion of this work was presented as an OPEN FORUM abstract at the American Association of Respiratory Care Congress, held October 15-18, 2016, in San Antonio, Texas.

Dr Khemani's institution received funding from National Institutes of Health (NIH)/National Institute of Child Health and Human Development (NICHD) 1K23HL103785 and from Los Angeles Basin Clinical Translational Science Institute. He received support for article research from the NIH. The other authors have disclosed no conflicts of interest.

Correspondence: Justin C Hotz RRT-NPS, Mailstop 12, Department of Anesthesiology and Critical Care, Children's Hospital Los Angeles, 4650 Sunset Boulevard, Los Angeles, CA 90027. E-mail: jhotz@chla.usc.edu.

DOI: $10.4187 /$ respcare. 05685

\section{QUICK LOOK}

\section{Current knowledge}

An approach to mechanical ventilation that uses transpulmonary pressure as a guide is promising in adults, but previous laboratory and clinical studies have demonstrated that inappropriately filled esophageal balloons can report erroneous measurements, and very little laboratory or clinical data exist in this area for pediatrics.

\section{What this paper contributes to our knowledge}

In a simulated pediatric esophagus, pediatric esophageal balloons overestimated surrounding pressure when using an inflation volume within the manufacturers' recommended ranges. Using an optimal filling volume that corresponded with the least rate of change in esophageal pressure per unit of filling volume assured accuracy in a laboratory study and was feasible to obtain in 5 critically ill pediatric subjects.

sure of chamber pressure $\left(\mathrm{P}_{\text {chamber }}\right)$. We subsequently tested the impact of balloon inflation volume on measured $\mathrm{P}_{\mathrm{L}}$ in pediatric subjects with ARDS and the feasibility of using our filling-volume technique in clinical practice. Finally, in the laboratory model, we evaluated the accuracy of liquid-filled catheters and fiberoptic transducers (microtip) under the same conditions.

Both the laboratory component and the research with human subjects were conducted at Children's Hospital Los Angeles, California. This work was supported, in part, by grants from National Institutes of Health/National Institute of Child Health and Human Development 1K23HL103785, and from Los Angeles Basin Clinical Translational Science Institute.

\section{Laboratory Study}

Experimental Set-up. A 4-L rigid plastic cylinder was connected to a controlled pressure source (Cough Assist, Philips Respironics, Murrysville, Pennsylvania). $\mathrm{P}_{\text {chamber }}$ was monitored via non-compliant pressure tubing connected to a pressure transducer (Bicore II, CareFusion, Houten, The Netherlands) (Fig. 1). Esophageal catheters were placed in the chamber through a small opening that was then sealed with putty. Each catheter was tested without coming in contact with the walls of the chamber (without a model esophagus) and after being inserted into a model esophagus, which consisted of a section of $6 \mathrm{~mm}$ inner diameter Penrose drain (Medline Industries, Mundelein, Illinois) covering the entire length of the esophageal balloon, or the distal tip of the catheter for 


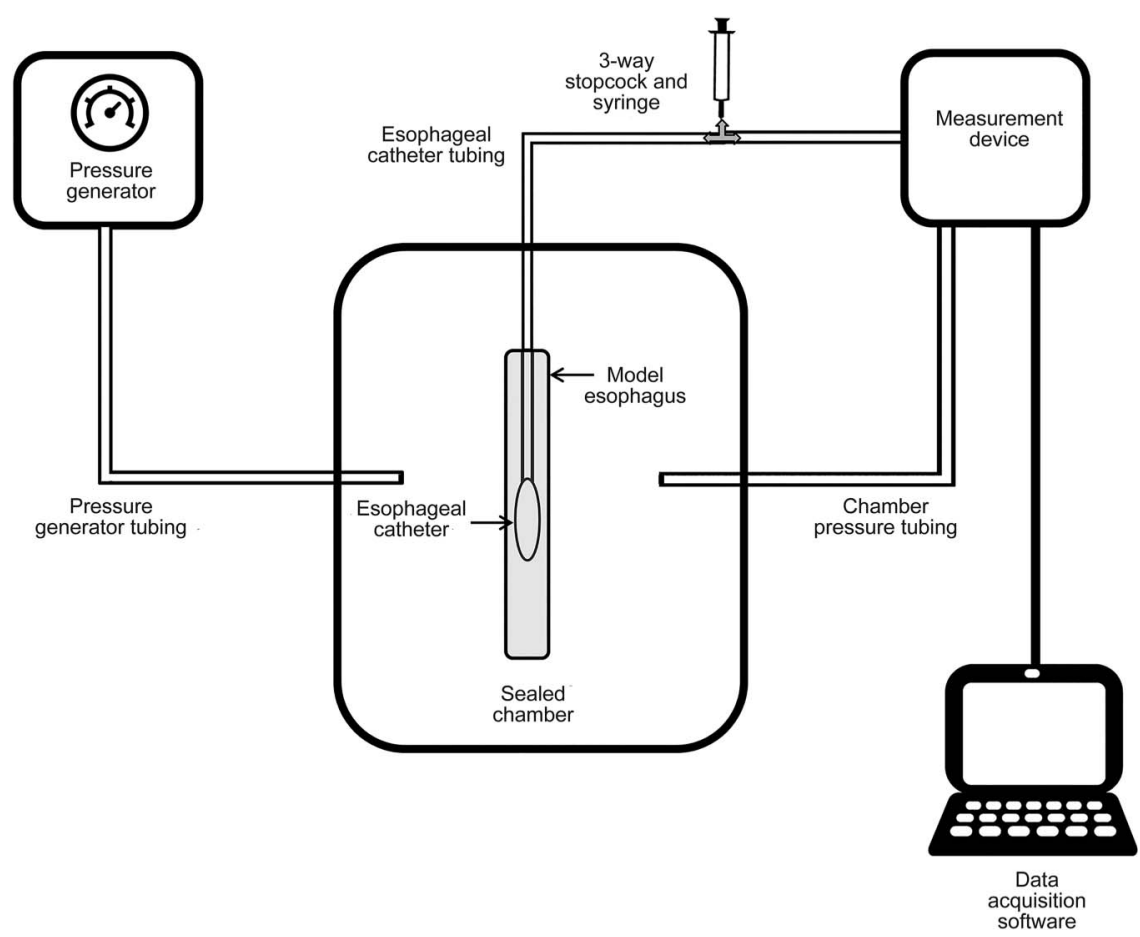

Fig. 1. The experimental chamber consisted of a rigid plastic cylinder that was pressurized to 5 different levels using a pressure generator. Each esophageal catheter was tested both with and without a model esophagus. Inflation of the esophageal balloon catheter was accomplished via a 3-way stop cock and pressure tubing. The experimental chamber and esophageal catheter were connected to a measurement device via pressure tubing.

the liquid-filled and micro-tip catheter. For each test, the chamber was pressurized at $5,10,15,20$, and $30 \mathrm{~cm}$ $\mathrm{H}_{2} \mathrm{O}$ via sustained 5-s inflations. Simultaneous $\mathrm{P}_{\text {chamber }}$ and $\mathrm{P}_{\mathrm{es}}$ were recorded at a frequency of $200 \mathrm{~Hz}$ on a laptop computer using a custom software module developed in Polybench (Applied Biosignals, GmbH, Weener, Germany). Experiments were repeated in triplicate, with a new catheter each time.

We studied 3 different catheter types: esophageal-balloon, liquid-filled, and micro-tuip transducer catheters. Four different esophageal balloon catheters were evaluated: 6 French and 7 French pediatric catheter and an 8 French adult SmartCath catheter (Viyaire Medical, Mettawa, Illinois), and a 5 French adult catheter from Cooper-Surgical (Trumbull, Connecticut). An 8 French liquid-filled CORFLO nasogastric tube (CORPAK MedSystems, Buffalo Grove, Illinois) and a fiberoptic micro-tip pressure transducer using a Camino ICP monitor (Integra, Plainsboro, New Jersey) were also evaluated.

Esophageal-Balloon Catheter Protocol. At each set $\mathrm{P}_{\text {chamber }}$ level, and with and without an esophagus, esophageal balloons were inflated in $0.1-\mathrm{mL}$ steps with a $1-\mathrm{mL}$ syringe for the pediatric catheters, or in $0.2-\mathrm{mL}$ steps with a 3-mL syringe for the adult catheters, to a maximum inflating volume of $1.0 \mathrm{~mL}$ air for the pediatric catheters and $2.0 \mathrm{~mL}$ air for the adult catheters. For each step, before in- flation, the esophageal balloon was deflated and subsequently allowed to equilibrate with atmospheric pressure for $10 \mathrm{~s}$.

Liquid-Filled Catheter Protocol. The nasogastric tube was inserted into the chamber, both with and without a model esophagus, connected to disposable tubing normally used for arterial blood pressure monitoring, and subsequently connected to a disposable blood-pressure transducer (Edwards Lifesciences, Irvine, California) and displayed on a Philips MP60 IntelliVue monitor (Philips Medical System, Best, The Netherlands). Prior to each measurement, the system was flushed with sterile water to ensure a patent column of water, and the catheter was zeroed to align with the blood-pressure transducer. Each cycle of measurements was repeated 3 times, and a new disposable transducer was used for each cycle. Finally, to simulate clinical conditions, the experiments were repeated with the catheter zeroed both $5 \mathrm{~cm}$ above and below the blood-pressure transducer.

Micro-Tip Pressure Transducer Protocol. The microtip pressure transducer from the Camino catheter was electronically zeroed to atmospheric pressure prior to being introduced to the chamber, both with and without a model esophagus. At each set $\mathrm{P}_{\text {chamber }}$, simultaneous $\mathrm{P}_{\mathrm{es}}$ measured from the Camino Advanced Monitor and 


\section{Measurements in Pediatric Esophageal Balloon Catheters}

the $\mathrm{P}_{\text {chamber }}$ were recorded, again repeating the experiment 3 times.

Data Analysis. For each testing sequence and condition, accuracy was assessed by the outcome variable, total error, defined as (measured value - true value $)^{14}$ or $\left(\mathrm{P}_{\mathrm{es}}-\right.$ $\left.\mathrm{P}_{\text {chamber }}\right)$. The mean and range of total error for each catheter and condition were reported, with positive values indicating that the pressure measured by the esophageal catheter is falsely high (overestimation of $\mathrm{P}_{\text {chamber }}$ ), and negative values indicating that the pressure measured by the esophageal catheter is falsely low (underestimation of $\mathrm{P}_{\text {chamber }}$ ).

\section{Derivation of the Optimal Filling-Volume Technique.} Because we noted significant inaccuracies in the $\mathrm{P}_{\mathrm{es}}$ as a function of balloon inflation volume, we tried to identify a method to determine the optimal filling volume under a given condition (catheter size, set $\mathrm{P}_{\text {chamber }}$ ) that could be translated to the bedside. To do this, we examined simultaneous plots of the balloon inflation volume versus $P_{e s}$, superimposed with the observed total error. We identified an inflection point or range on the curve of inflation volume and $\mathrm{P}_{\mathrm{es}}$ that resulted in the most accurate $\mathrm{P}_{\text {chamber }}$ estimates. We then derived a method to identify this inflection point that did not require graphical analysis.

\section{Clinical Study}

We conducted a feasibility pilot study to determine the effect that manipulating esophageal balloon filling volume would have on $\mathrm{P}_{\mathrm{es}}$ and respective calculated $\mathrm{P}_{\mathrm{L}}$ in mechanically ventilated pediatric subjects. This study was conducted in a subset of subjects enrolled in an ongoing clinical investigation of mechanically ventilated children ( $<18$ y old) with ARDS ventilated with pressure-controlled intermittent mandatory ventilation in which both $\mathrm{P}_{\mathrm{L}}$ and effort of breathing calculations were being obtained. Inclusion criteria were an anticipated length of intubation $>48 \mathrm{~h}$ with an oxygen saturation index ${ }^{15}$ $\left(100 \times \overline{\mathrm{P}}_{\mathrm{aw}} \times \mathrm{F}_{\mathrm{IO}_{2}} / \mathrm{S}_{\mathrm{pO}_{2}}\right)>=5$ to meet oxygenation criteria for pediatric ARDS. ${ }^{16}$ Patients were excluded if they had a corrected gestational age $<37$ weeks, contraindications to nasoesophageal catheter placement (nasopharyngeal or esophageal abnormalities), or had significant lower airway obstruction. On each study day, the esophageal catheter position was confirmed by chest radiograph. The protocol was approved by the CHLA Institutional Review Board, and informed consent was obtained from each child's parent or guardian.

Study Protocol. All the subjects were intubated and mechanically ventilated with either an Avea or Servo-i ventilator and had an age-appropriate SmartCath esophageal balloon catheter placed prior to the study. Esophageal pressure was measured with the Bicore II as described in the laboratory experiment. $\mathrm{P}_{\mathrm{aw}}$ was measured with the ventilator. Subjects were studied in the supine position. Ventilator settings were managed by the medical team. Once daily, $\mathrm{P}_{\mathrm{es}}$ and $\mathrm{P}_{\mathrm{aw}}$ were recorded over the range of esophageal balloon inflation levels as described in the laboratory experiment: from a volume of $0.1-1.0 \mathrm{~mL}$ in $0.1-\mathrm{mL}$ steps for the pediatric catheters, and $0.2-2.0 \mathrm{~mL}$ in $0.2-\mathrm{mL}$ steps for the adult catheters. Prior to each new inflation level, the esophageal balloon was deflated and allowed to equilibrate to atmospheric pressure for $10 \mathrm{~s}$. The $\mathrm{P}_{\mathrm{es}}$ signal was recorded at $200 \mathrm{~Hz}$ in the same fashion as described in the laboratory experiment and stored for analysis. Daily measurements were obtained between Monday and Friday until the subjects entered the weaning phase of ventilation and were consistently breathing spontaneously with a supported mode of ventilation.

Data Analysis. $\mathrm{P}_{\mathrm{L}}\left(\mathrm{P}_{\mathrm{aw}}-\mathrm{P}_{\mathrm{es}}\right)$ was measured during exhalation immediately before inspiration $\left(\mathrm{P}_{\text {Lexp }}\right)$, and during inhalation immediately before exhalation $\left(\mathrm{P}_{\text {Linsp }}\right)$, without a specific inspiratory or expiratory hold. $\mathrm{P}_{\text {Lexp }}$ and $\mathrm{P}_{\text {Linsp }}$ were determined for each subject, study day, and esophageal balloon inflation volume. The average of 3 values obtained from consistent and consecutive waveforms was used in calculations for each inflation level.

\section{Results}

\section{Laboratory Study}

Esophageal Balloon Catheters. The mean and range total error of the esophageal balloon catheters as a function of esophageal balloon inflation volume is shown for all conditions tested in Figure 2. In all cases, a small volume of air was required to avoid underestimating $\mathrm{P}_{\text {chamber }}$. When not using a model esophagus, the measured $\mathrm{P}_{\mathrm{es}}$ (and corresponding $\mathrm{P}_{\mathrm{es}}-\mathrm{P}_{\text {chamber }}$ difference) changes by a small amount when air is added in increasing amounts to the esophageal balloon. This resulted in minimal total error across the entire manufacturer-recommended range (0.5-1.25 $\mathrm{mL}$ for the 6 French and 7 French Pediatric CareFusion Catheter, $0.5-2.25 \mathrm{~mL}$ for the 8 French Adult CareFusion Catheter, and $0.5-1.0 \mathrm{~mL}$ for the 5 French Adult Cooper Catheter). When a model esophagus was used, there was a more rapid change in $\mathrm{P}_{\mathrm{es}}$ when the same amount of air is added to the balloon. This results in a larger degree of total error and overestimation of $\mathrm{P}_{\text {chamber }}$ as a function of increasing inflation volume, even within the manufacturer's suggested range, particularly for the pediatric esophageal balloons. The total error as a function of inflation volume is most pronounced when $\mathrm{P}_{\text {chamber }}$ is lowest (ie, $5 \mathrm{~cm} \mathrm{H}_{2} \mathrm{O}$ ). 


\section{Measurements in Pediatric Esophageal Balloon Catheters}

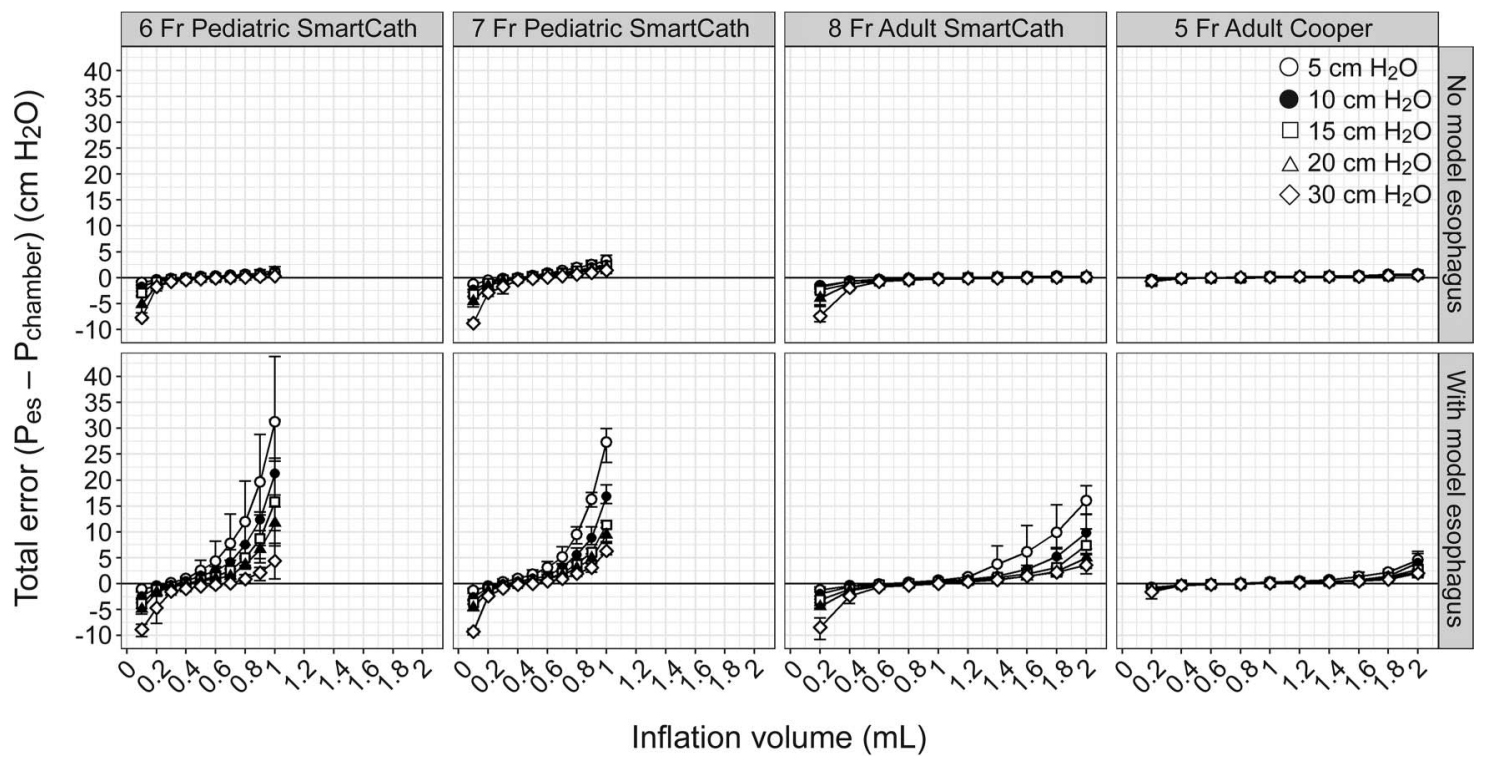

Fig. 2. The mean and range (error bars) for total error of the esophageal balloon catheters as a function of esophageal balloon inflation volume, and set chamber pressure $\left(\mathrm{P}_{\text {chamber }} 5,10,15,20\right.$, or $\left.30 \mathrm{~cm} \mathrm{H}_{2} \mathrm{O}\right)$ stratified by the presence and absence of a model esophagus, and catheter type.

Optimal Filling Volume. Because there was an overestimation of esophageal pressure with larger inflation volumes, which is also dependent on $\mathrm{P}_{\text {chamber }}$, a single inflation amount based on catheter size is unlikely to yield consistently accurate measures of $\mathrm{P}_{\mathrm{es}}$. When reviewing the $\mathrm{P}_{\mathrm{es}}$-inflation volume curves, 2-3 different phases were observed as inflation volume is incrementally increased, first a rapid climb in $\mathrm{P}_{\mathrm{e}}$, which is likely the pressurization of air inside the catheter; second, a more gradual increase in $\mathrm{P}_{\mathrm{es}}$, likely due to inflation of the esophageal balloon; and, in some cases, a third phase when the balloon is overdistended and there is another rapid increase in $\mathrm{P}_{\mathrm{es}}$ (Fig. 2). The region with the least amount of total error tended to be immediately at the beginning of the second phase, after the catheter is pressurized and the balloon is just beginning to inflate, where there is a gradual climb in $\mathrm{P}_{\mathrm{es}}$ as inflation volume is increased. This region is characterized as having lower elastance (lower change in pressure per unit change in volume), thus at each inflation volume we calculated the elastance of the esophageal balloon system as $\mathrm{E}_{\text {system }}=\Delta \mathrm{P}_{\mathrm{es}} / \Delta \mathrm{V}$, where $\Delta \mathrm{P}_{\mathrm{es}}=\left(\mathrm{P}_{\mathrm{es}}\right.$ at $\left.\mathrm{V}+\right)-\left(\mathrm{P}_{\mathrm{es}}\right.$ at $\mathrm{V}-$ ), and $\Delta \mathrm{V}=\mathrm{V}+-\mathrm{V}-$, which are the inflation volumes immediately above and below the inflation volume at which $\mathrm{E}_{\text {system }}$ is being calculated. Because the $\Delta \mathrm{V}$ is constant at either $0.2 \mathrm{~mL}$ for the pediatric balloon or $0.4 \mathrm{~mL}$ for the adult balloon, we can simply report $\Delta \mathrm{P}_{\mathrm{es}}$ as the metric to refer to the relative change in $\mathrm{E}_{\text {system }}$ at each inflation volume. After calculating $\Delta \mathrm{P}_{\mathrm{es}}$ for all inflation volumes, we could infer the inflation volume at the beginning of this lower elastance region by discarding the top half of inflation volumes that had the highest values for
$\Delta \mathrm{P}_{\mathrm{es}}$ and using the lowest inflation volume that was remaining (see the supplementary materials 1 and 2 at http://www.rcjournal.com). Simply using the inflation volume with the lowest $\Delta \mathrm{P}_{\mathrm{es}}$ may provide higher total error for cases where there is a wide inflation volume range with low elastance, such as when using an adult esophageal balloon catheter and the lowest $\mathrm{E}_{\text {system }}$ for a given inflation happens to occur at the higher end of this region.

We find that this technique identifies the beginning of the second phase, or the lower elastance region, and varies based on the surrounding $\mathrm{P}_{\text {chamber }}$ and catheter type (Fig. 3 ). The mean (and range) optimal filling volumes derived for the pediatric esophageal balloon catheters when using a model esophagus was $0.3(0.2-0.6) \mathrm{mL}$, and $0.6(0.4-$ $0.8) \mathrm{mL}$ for the adult esophageal balloon catheters. The mean total error and precision (95\% CI) between esophageal and chamber pressures when using the derived optimal filling technique, using Bland-Altman plots for all conditions, are shown in Figure 4. When using a filling volume that corresponds with optimal filling volume for each catheter, pressure level, and test sequence, the mean total error ranges from -0.53 to $-0.10 \mathrm{~cm} \mathrm{H}_{2} \mathrm{O}$, which is greatly improved over the range total error obtained from the manufacturer-recommended range with the smaller pediatric catheters. (To aid in simplicity of calculation at the bedside, we have developed an R Shiny web application that requires the user to enter just the value for $\mathrm{P}_{\mathrm{es}}$ obtained at each inflation volume, and it will compute the optimal filling volume: https://justinhotz.shinyapps.io/ EsophagealBalloonApp/. (The source code for the appli- 


\section{Measurements in Pediatric Esophageal Balloon Catheters}

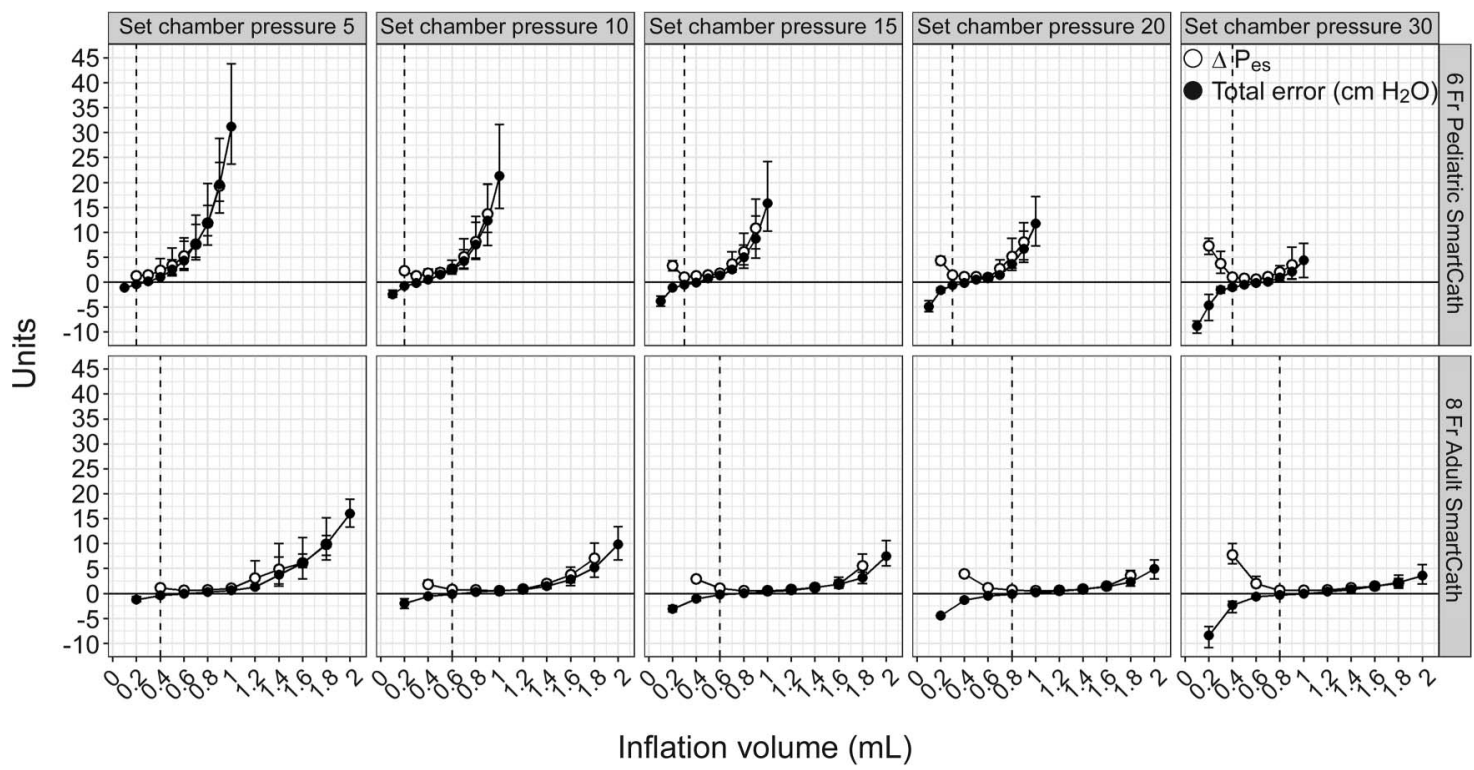

Fig. 3. Mean and range (error bars) for esophageal pressure $\left(P_{e s}\right)$ and total error of the esophageal balloon catheters as a function of esophageal balloon inflation volume and set chamber pressure are shown for the $6 \mathrm{Fr}$ Pediatric (manufacturer-recommended range 0.5-1.25 mL) and $8 \mathrm{Fr}$ Adult (manufacturer-recommended range $0.5-2.25 \mathrm{~mL}$ ) CareFusion catheters when a model esophagus is used. The dotted vertical lines show the inflation volume that corresponds with the derived optimal filling volume (the median of 3 tests is shown). Note that the optimal filling volume technique commonly results in no difference between chamber and measured esophageal pressure (total error near 0 ), but there is a tendency to overestimate $P_{\text {chamber }}$ when using the higher end of the manufacturer-recommended range.

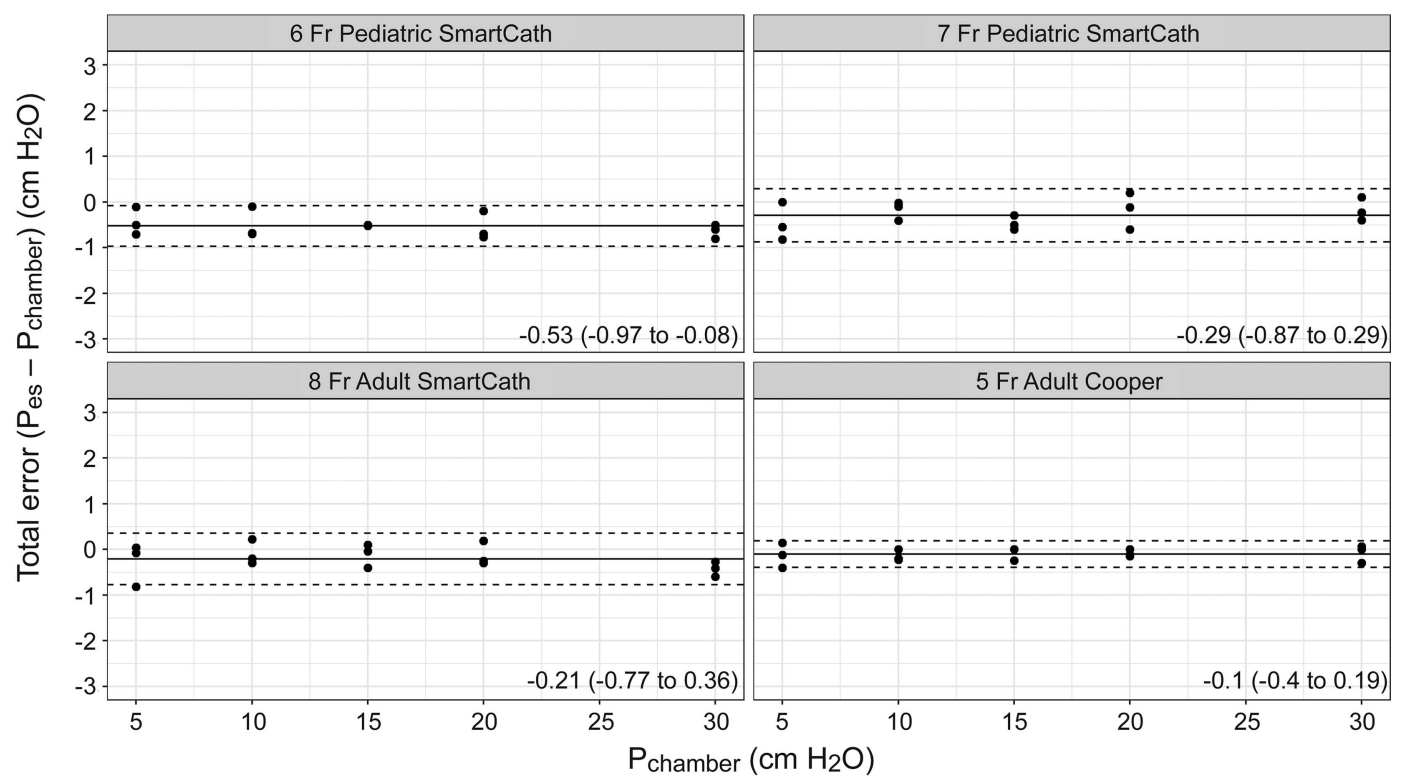

Fig. 4. Mean total error and precision $(95 \% \mathrm{Cl})$ is shown with Bland-Altman plots when using a model esophagus and an esophageal balloon inflation volume that corresponds with the optimal filling volume for each testing condition. Results are stratified by catheter type.

cation is available at https://github.com/JustinHotz/ EsophagealBalloonApplication.)

\section{Liquid-Filled and Micro-Tip Pressure Transducer.} Both the liquid-filled and the micro-tip transducer maintained high accuracy across the range of pressures, regardless of whether a model esophagus was used (Fig. 5). The mean total error is $\pm 1 \mathrm{~cm} \mathrm{H}_{2} \mathrm{O}$ at all chamber pressure levels when using the Camino Catheter, or when the liquid-filled catheter tip is appropriately zeroed with the transducer. When the liquid-filled catheter opening is $5 \mathrm{~cm}$ above or below the transducer, the chamber pressure has a predictable over- or underestimation close to $5 \mathrm{~cm} \mathrm{H}_{2} \mathrm{O}$ at all chamber pressure levels. 


\section{Measurements in Pediatric Esophageal Balloon Catheters}

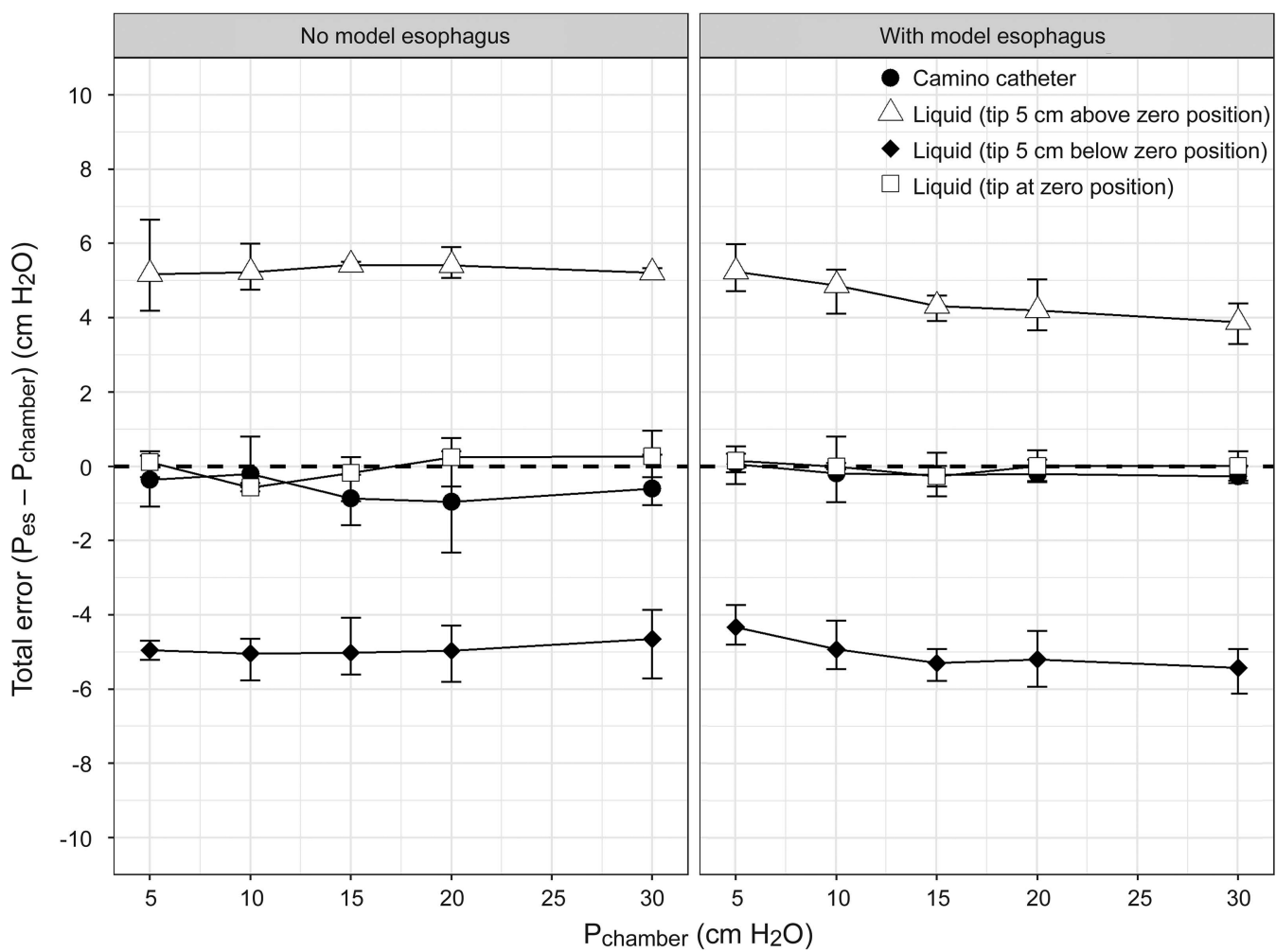

Fig. 5. Mean and range (error bars) difference between chamber $\left(P_{\text {chamber }}\right)$ and measured esophageal pressure $\left(P_{\text {es }}\right)$ is shown for the Camino catheter (micro-tip transducer) and the liquid-filled catheter (8 Fr CORFLO) when the liquid-filled catheter tip is level with the transducer and both $5 \mathrm{~cm}$ above and below the transducer, stratified by the presence and absence of a model esophagus.

\section{Clinical Study}

Five subjects were included in the analysis for the clinical study. Only the 6 French CareFusion Pediatric Catheter and the 8 French CareFusion Adult Catheter were used for the clinical study. A total of 9 distinct measurement days of data were obtained. Upon reviewing chest radiographs, the catheter was not in an appropriate position for 2 of the measurements, and results for these 2 cases can be found online (see the supplementary materials 3 at http://www.rcjournal.com). For the 7 measurements in which the catheter was in an appropriate position, demographic and clinical features including age, derived optimal filling volumes, and corresponding $\mathrm{P}_{\text {Lexp }}$ and $\mathrm{P}_{\text {Linsp }}$ when using the optimal filling volume, as well as the minimum and maximum values for the manufacturers' recommended ranges, are presented in Table 1 for each subject and distinct measurement day. The mean and range of inflation volumes obtained for optimal filling volume for the 6 French CareFusion Pediatric Catheter and the 8 French CareFusion Adult Catheter were $0.4(0.2-0.5) \mathrm{mL}$ and 0.8 $(0.6-1.0) \mathrm{mL}$, respectively. When aggregating subject measurements together, Figure 6 shows the measured $\mathrm{P}_{\text {Lexp }}$ and $\mathrm{P}_{\text {Linsp }}$ (median, IQR) for each esophageal balloon inflation volume and catheter type. When using the manu- facturer-recommended range, for the 6 French Carefusion Pediatric Catheter the median values for $\mathrm{P}_{\text {Lexp }}$ on clinician selected ventilator settings range from -6.5 to $-2.5 \mathrm{~cm}$ $\mathrm{H}_{2} \mathrm{O}$, and $\mathrm{P}_{\text {Linsp }}$ range from 7.0 to $9.8 \mathrm{~cm} \mathrm{H}_{2} \mathrm{O}$. Using the optimal filling volume technique, this range is narrower (filling volume $0.2-0.5 \mathrm{~mL}$ ) and higher with median $\mathrm{P}_{\text {Lexp }}$ ranging from 0.0 to $-2.5 \mathrm{~cm} \mathrm{H}_{2} \mathrm{O}$ and $\mathrm{P}_{\text {Linsp }}$ ranging from 9.8 to $12.0 \mathrm{~cm} \mathrm{H}_{2} \mathrm{O}$. For the 8 French CareFusion Adult Catheter, the manufacturer's range yielded median for $\mathrm{P}_{\text {Lexp }}$ values ranging from -3.6 to $1.0 \mathrm{~cm} \mathrm{H}_{2} \mathrm{O}$ and $\mathrm{P}_{\text {Linsp }}$ values ranging from 13.7 to $18.7 \mathrm{~cm} \mathrm{H}_{2} \mathrm{O}$. The optimal filling volume technique further narrowed this range (filling volume $0.6-1.0 \mathrm{~mL}$ ) to be from -1.0 to $1.0 \mathrm{~cm} \mathrm{H}_{2} \mathrm{O}$ for $\mathrm{P}_{\text {Lexp }}$ and from 17.6 to $18.6 \mathrm{~cm} \mathrm{H}_{2} \mathrm{O}$ for $\mathrm{P}_{\text {Linsp. }}$.

\section{Discussion}

We have demonstrated in a combination bench and clinical study that esophageal pressure measurements with balloon catheters may be very inaccurate if balloon inflation volume is not correct. The accuracy of pediatric balloon catheters is especially vulnerable to inflation volume, even within the manufacturer's recommended limits. Some of these limitations can be overcome if the optimal balloon inflation volume is identified, and we have suggested a 


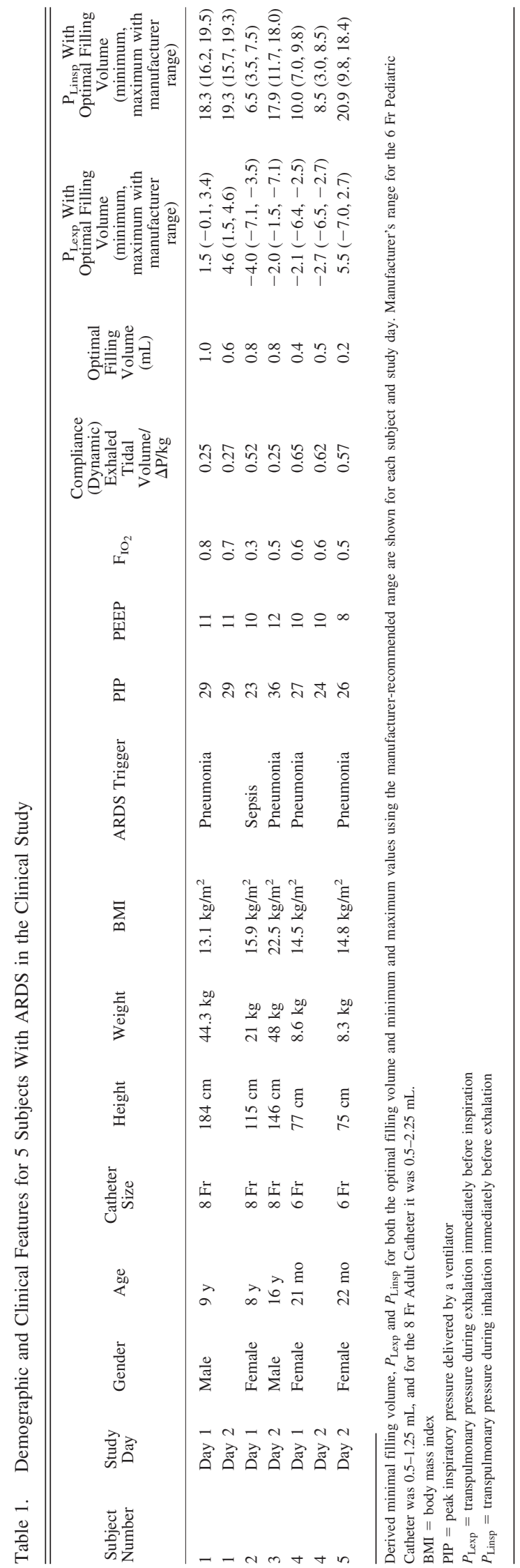

method to determine this volume at the bedside for an individual patient, at a given point in time, with an individual catheter. Nevertheless, other techniques such as liquid-filled or micro-tip catheters may be more promising, because they appear to maintain accuracy over a wider range of $\mathrm{P}_{\mathrm{es}}$ without being affected by the elastance of the esophagus.

From the laboratory portion of our study, we found that a model esophagus was crucial to simulate real-world conditions, as the overestimation of chamber pressure from the esophageal balloons within the manufacturer's range is only present with the model esophagus. We believe this simulates clinical conditions, and the differences we observed in measured esophageal pressure as a function of inflation volume in subjects correspond well to the model esophagus scenario. Our experimental set-up and results without a model esophagus were similar to 2 laboratory studies performed by Mojoli et al ${ }^{11}$ and Walterspacher et $\mathrm{al}^{12}$ who found a relatively wide range of esophageal balloon filling volumes that would yield a similar value for $\mathrm{P}_{\mathrm{es}}$, indicating a wide working range with low elastance. Walterspacher et al observed that this working range could change due to material adhesion characteristics of the esophageal balloon both immediately after the first inflation and also after being exposed to large inflation volumes for $16 \mathrm{~h}$, indicating that the working range even without a model esophagus may change over time. ${ }^{12}$ While performing a clinical study in 36 adults, Mojoli et al ${ }^{13}$ observed a steeper pressure-volume relationship than they had previously reported in their laboratory study, and they reasoned that the added elastance of the esophagus contributed to a falsely high value for $\mathrm{P}_{\mathrm{es}}$. Indeed, the added elastance of the esophageal wall has been cited as a potential source of error when measuring $\mathrm{P}_{\mathrm{es}}{ }^{2,11-13,17-19}$ To compensate for this, Mojoli et al ${ }^{13}$ proposed performing an in vivo calibration to characterize the individual elastance of each subject's esophagus. This method was tested with one brand of adult esophageal balloon and required visual inspection to identify the linear portion of the $\mathrm{P}_{\mathrm{es}}$-inflation volume curve. This reinforces why a model esophagus is necessary to better simulate the clinical environment.

Similar to the method proposed by Mojoli et al, ${ }^{13}$ we too identified an in vivo calibration technique to determine the optimal filling volume for pediatric and adult esophageal balloon catheters. To potentially automate the visual technique described by Mojoli et al, ${ }^{13}$ we derived an optimal filling volume technique, which can be done at the bedside without graphing the data. The technique requires only the ability to view the recorded $\mathrm{P}_{\mathrm{es}}$ waveforms, either with recording software or the front-end of a ventilator with a $\mathrm{P}_{\mathrm{es}}$ waveform display, and documenting the $\mathrm{P}_{\mathrm{es}}$ at end exhalation as esophageal balloon inflation volume is increased in uniform amounts to perform the calculation for optimal filling volume (see the supplementary materials 1 


\section{Measurements in Pediatric Esophageal Balloon Catheters}

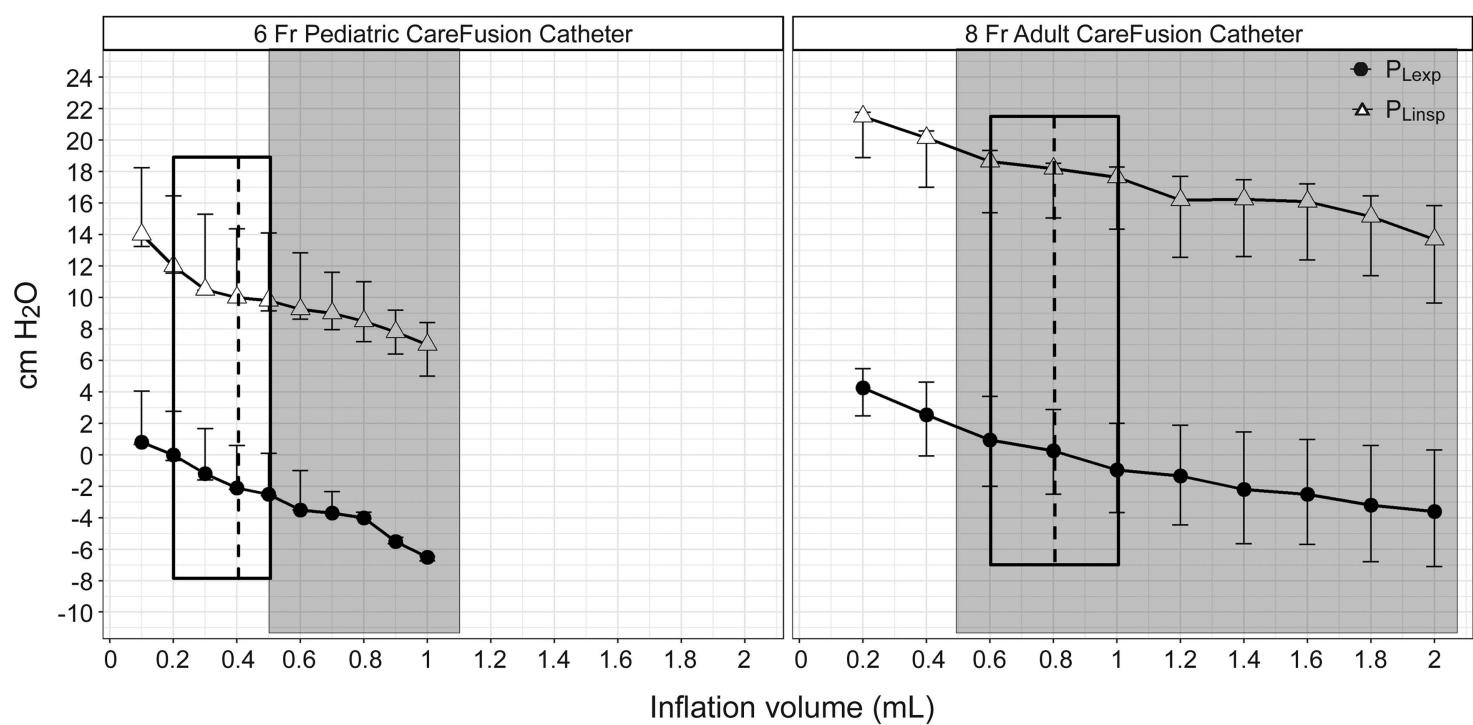

Fig. 6. Median and IQR (error bars) for $P_{\text {Lexp }}$ and $P_{\text {Linsp }}$ are shown for subjects in the clinical study as a function of esophageal balloon filling volume, stratified by catheter size. Shaded regions highlight the manufacturer-recommended range, and dotted lines and black outline show the mean and range of the derived minimal filling volumes obtained.

at http://www.rcjournal.com) or by using our web-based application. To our knowledge, ours is the first work published on the relationship between pediatric esophageal balloon inflation volume and $\mathrm{P}_{\mathrm{es}}$. While not the same in diameter or material, some extrapolation may be considered from work done by Mead et $\mathrm{al}^{20}$ and Petit and MilicEmili $^{21}$ in the 1950 s, who observed greater changes in measured esophageal pressure in relation to step-wise increases in esophageal balloon filling volume when using a short versus long esophageal balloon. They observed steeper slopes of $\mathrm{P}_{\mathrm{es}}$ versus inflation volume curves when using smaller esophageal balloons, and Petit and MilicEmili ${ }^{21}$ reasoned that the strain on the esophageal walls per unit of volume was greater with the smaller balloons because the longer balloons allowed air volume to be distributed over a longer segment of the esophagus. Pediatric esophageal balloon catheters are smaller in diameter and length than adult esophageal balloon catheters, and their working range where accuracy can be assured may be smaller. This puts them at much higher risk of overestimating pleural pressure than adult balloons, which are longer and wider.

To that end, using the pediatric esophageal balloon catheters, our optimal filling volume technique requires considerable precision and can be prone to error as the low elastance region of the $\mathrm{P}_{\mathrm{es}}$-inflation volume curve may be very small $(<0.3 \mathrm{~mL})$, particularly when the actual pleural pressure is low (Fig. 3). For small children, it may be beneficial to consider an alternative system of esophageal pressure measurements, such as a liquid-filled system or a micro-tip transducer, where the $\mathrm{P}_{\mathrm{es}}$-inflation volume relationship of the esophageal balloon is negated. Liquid-filled catheters can be as simple as a standard feeding tube that is filled with a continuous low-flow infusion of liquid that transmits pressure from the tip of the catheter to a transducer. We demonstrated good accuracy using the liquidfilled catheter, although it will over- or underestimate pressure if the column of liquid is not patent or the tip is not level with the transducer. This zeroing of pressure may be difficult to achieve during clinical care, where body position may be adjusted frequently. ${ }^{22-24}$ The micro-tip transducer allows $\mathrm{P}_{\mathrm{es}}$ to be measured directly at the catheter tip $^{25}$ and was accurate across all conditions tested, regardless of whether a model esophagus was used, but it may drift over time, which would require intermittent zeroing 26 and thus makes it less practical for long-term ventilator management. Further innovation is necessary to provide a reliable $\mathrm{P}_{\mathrm{es}}$ measurement system for children that is not dependent on balloon inflation or body position, and is able to maintain calibration throughout the course of mechanical ventilation.

Our study has a number of limitations. First, the laboratory portion of the study utilized a Penrose drain to simulate an esophagus, which is an oversimplification for a biologic specimen and may not yield a realistic pediatric esophageal elastance. Second, we used the same size Penrose drain for both the pediatric and adult esophageal balloon catheters, which may provide a falsely high elastance for the adult esophageal balloons. However, it is clear that a model esophagus is necessary to more closely mimic clinical conditions, and so a model esophagus should be included in future bench models to test esophageal balloon catheters. Third, the laboratory experiment did not use added heat and humidity in the chamber, which may cause 


\section{Measurements in Pediatric Esophageal Balloon Catheters}

the instilled gas in the balloon to expand further and provide potentially greater pressure overestimation than what was reported. Fourth, the clinical study was a small sample size; this work should be repeated with a larger sample size to confirm findings. Fifth, the $\mathrm{P}_{\mathrm{L}}$ measurements did not use inspiratory and expiratory holds, which may provide different values than when a hold is performed, although this would predominately affect $\mathrm{P}_{\mathrm{aw}}$ and $\mathrm{P}_{\mathrm{es}}$ values and should not change significantly with or without a hold (see the supplementary materials 4 at http://www.rcjournal. com).

\section{Conclusions}

We have demonstrated that $\mathrm{P}_{\mathrm{es}}$ is highly dependent on esophageal balloon inflation volume, even within the manufacturer's recommended limits. The pediatric esophageal balloon catheter has a smaller working range of balloon inflation volume than the adult-sized esophageal balloon catheter, although both should undergo an in vivo calibration method to determine optimal inflation volume. We have derived a method that may provide a good estimate for the inflation volume that is required to assure better accuracy. Other means for estimating esophageal pressure that are less subject to error are needed for pediatric patients.

\section{ACKNOWLEDGMENT}

We would like to acknowledge Dr. Jan Kelly, Children's Hospital Zürich and Professor Peter Rimensberger, University Hospital of Geneva for initially drawing our attention to the particular problems of esophageal balloon catheters in infants and children.

\section{REFERENCES}

1. Loring SH, Topulos GP, Hubmayr RD. Transpulmonary pressure: the importance of precise definitions and limiting assumptions. Am J Respir Crit Care Med 2016;194(12):1452-1457.

2. Sahetya SK, Brower RG. The promises and problems of transpulmonary pressure measurements in acute respiratory distress syndrome. Curr Opin Crit Care 2016;22(1):1-13.

3. Talmor D, Sarge T, Malhotra A, O'Donnell CR, Ritz R, Lisbon A, et al. Mechanical ventilation guided by esophageal pressure in acute lung injury. New Engl J Medicine 2008;359(20):2095-2104.

4. Chiumello D, Cressoni M, Colombo A, Babini G, Brioni M, Crimella $\mathrm{F}$, et al. The assessment of transpulmonary pressure in mechanically ventilated ARDS patients. Intensive Care Med 2014; 40(11):1670-1678.

5. Terragni P, Filippini C, Slutsky A, Birocco A, Tenaglia T, Grasso S, et al. Accuracy of plateau pressure and stress index to identify injurious ventilation in patients with acute respiratory distress syndrome. Anesthesiology 2013;119(4):880-889.

6. Grasso S, Terragni P, Birocco A, Urbino R, Del Sorbo L, Filippini $\mathrm{C}$, et al. ECMO criteria for influenza A (H1N1)-associated ARDS: role of transpulmonary pressure. Intensive Care Med 2012;38(3): 395-403.

7. Chiumello D, Carlesso E, Cadringher P, Caironi P, Valenza F, Polli $\mathrm{F}$, et al. Lung stress and strain during mechanical ventilation for acute respiratory distress syndrome. Am J Respir Crit Care Med 2008;178(4):346-355.

8. Akoumianaki E, Maggiore SM, Valenza F, Bellani G, Jubran A, Loring $\mathrm{SH}$, et al. The application of esophageal pressure measurement in patients with respiratory failure. Am J Respir Crit Care Med 2014;189(5):520-531.

9. Crotti S, Mascheroni D, Caironi P, Pelosi P, Ronzoni G, Mondino M, et al. Recruitment and derecruitment during acute respiratory failure: a clinical study. Am J Respir Crit Care Med 2001;164(1):131-140.

10. Dechman G, Sato J, Bates JH. Factors affecting the accuracy of esophageal balloon measurement of pleural pressure in dogs. J Appl Physiol 1992;72(1):383-388.

11. Mojoli F, Chiumello D, Pozzi M, Algieri I, Bianzina S, Luoni S, et al. Esophageal pressure measurements under different conditions of intrathoracic pressure: An in vitro study of second generation balloon catheters. Minerva Anestesiol 2015;81(8):855-864.

12. Walterspacher S, Isaak L, Guttmann J, Kabitz H-J, Schumann S. Assessing respiratory function depends on mechanical characteristics of balloon catheters. Respir Care 2013;59(9):1345-1352.

13. Mojoli F, Iotti GA, Torriglia F, Pozzi M, Volta CA, Bianzina S, et al. In vivo calibration of esophageal pressure in the mechanically ventilated patient makes measurements reliable. Crit Care 2016;20: 98.

14. Chatburn RL. Evaluation of instrument error and method agreement. AANA J 1996;64(3):261-268.

15. Khemani RG, Thomas NJ, Venkatachalam V, Scimeme JP, Berutti $\mathrm{T}$, Schneider JB, et al. Comparison of $\mathrm{SpO} 2$ to $\mathrm{PaO} 2$ based markers of lung disease severity for children with acute lung injury. Crit Care Med 2012;40(4):1309-1316.

16. The Pediatric Acute Lung Injury Consensus Conference Group. Pediatric acute respiratory distress syndrome: consensus recommendations from the Pediatric Acute Lung Injury Consensus Conference. Pediatr Crit Care Med 2015;16(5):428-439.

17. Hedenstierna G. Esophageal pressure: benefit and limitations. Minerva Anestesiol 2012;78(8):959-966.

18. Guerin C, Richard J-C. Comparison of two correction methods for absolute values of esophageal pressure in patients with acute hypoxemic respiratory failure, mechanically ventilated in intensive care unit. Respir Care. 2012;57(12):2045-2051.

19. Talmor D, Sarge T, O'Donnell CR, Ritz R, Malhotra A, Lisbon A, Loring SH. Esophageal and transpulmonary pressures in acute respiratory failure. Crit Care Med 2006;34(5):1389-1394.

20. Mead J, McIlroy MB, Selverstonez J, Kriete BC. Measurement of intraesophageal pressure. J Appl Physiol 1955;7(5):491-495.

21. Petit J, Milic-Emili G. Measurment of endoesophageal pressure. J Appl Physiol 1958;13(3):481-485.

22. Milic-Emili G, Petit JM. Relationship between endoesophageal and intrathoracic pressure variations in dog. J Appl Physiol 1959;14(4): 535-537.

23. Coates AL, Davis GM, Vallinis P, Outerbridge EW. Liquid-filled esophageal catheter for measuring pleural pressure in preterm neonates. J Appl Physiol 1989;67(2):889-893.

24. Beda A, Güldner A, Carvalho AR, Zin WA, Carvalho NC, Huhle R, et al. Liquid- and air-filled catheters without balloon as an alternative to the air-filled balloon catheter for measurement of esophageal pressure. PLoS One 2014;9(9):e103057.

25. Essouri S, Durand P, Chevret L, Balu L, Devictor D, Fauroux B, Tissières $\mathrm{P}$. Optimal level of nasal continuous positive airway pressure in severe viral bronchiolitis. Intensive Care Med 2011;37(12): 2002-2007.

26. Piper I, Barnes A, Smith D, Dunn L. The Camino intracranial pressure sensor: is it optimal technology? An internal audit with a review of current intracranial pressure monitoring technologies. Neurosurgery 2001;49(5):1158-1165. 